\title{
Integral Transforms and Semigroups of Linear Operators on the $W^{2}$-space
}

\author{
Katsuo TAKano*
}

Introduction. Let $W^{2}$ be the totality of measurable functions such that

$$
\int_{-\infty}^{\infty} \frac{|f(v)|^{2}}{v^{2}+1} d v<\infty
$$

When $f$ and $g$ are in $W^{2}$ we define the inner product of $f$ and $g$ by

$$
(f, g)=\int_{-\infty}^{\infty} f(v) \overline{g(v)} \frac{d v}{v^{2}+1} .
$$

Introducing the operations of addition and scalar multiplication in $W^{2}$ as usual, we can show that the space $W^{2}$ is the Hilbert space with norm $\|f\|=[(f, f)]^{1 / 2}$. Let

$$
p_{\lambda}(t, u)=\frac{\lambda t}{\pi\left[(\lambda t)^{2}+u^{2}\right]} .
$$

If $t>0$ and $\lambda>0$, this function $p_{\lambda}(t, u)$ is the Cauchy density function. When $\lambda=r-i q(r>0)$ and $t>0$ let

$$
\left(P_{\lambda}(t) f\right)(v)=\int_{-\infty}^{\infty} p_{\lambda}(t, u) f(v-u) d u
$$

and

$$
\left(P_{\lambda}(0) f\right)(v)=f(v)
$$

for $f$ in $W^{2}$. The purpose of this paper is to show that a family $\left\{P_{\lambda}(t): 0 \leqq t<\infty\right\}$ of linear operators on $W^{2}$ is a semigroup of class $\left(C_{0}\right)$ and that there exists a oneparameter strongly continuous group of linear operators $\left\{P_{-i q}(t):-\infty<t<\infty\right\}$ on $W^{2}$.

1. Semigroups and groups of linear operators on $W^{2}$. If $\lambda=r-i q(r>0)$ and $t>0$,

$$
\left|p_{\lambda}(t, u)\right|=\frac{t\left(r^{2}+q^{2}\right)^{1 / 2}}{\pi\left[\left(u^{2}+t^{2}\left(r^{2}-q^{2}\right)\right)^{2}+4 t^{4} r^{2} q^{2}\right]^{1 / 2}} .
$$

LEMMA 1.1. Let $\lambda=r-i q(r>0)$ and $t>0$. If $f$ is in $W^{2}$, then $\left(P_{\lambda}(t) f\right)(v)$

Received February 16, 1978.

* Department of Mathematics, College of General Education, Ibaraki University, Mito, Japan. 
is a function in $W^{2}$.

Proof. Take a positive number a such that $\left|t^{2}\left(r^{2}-q^{2}\right)\right|<a^{2}$. Then

$$
\begin{aligned}
\left|\left(P_{\lambda}(t) f\right)(v)\right| \leqq & \int_{-a}^{a}\left|p_{\lambda}(t, u) f(v-u)\right| d u+\int_{a}^{\infty}\left|p_{\lambda}(t, u) f(v-u)\right| d u \\
& +\int_{-\infty}^{-a}\left|p_{\lambda}(t, u) f(v-u)\right| d u .
\end{aligned}
$$

Since $\quad\left|p_{\lambda}(t, u)\right| \leqq K_{1}(r, q, t)<\infty \quad$ for $|u| \leqq a$ and $\left|p_{\lambda}(t, u)\right| \leqq K_{2}(r, q, t) u^{-2}$ for $|u|>a$, we have

$$
\begin{aligned}
& \left|\left(P_{\lambda}(t) f\right)(v)\right| \leqq K_{1}(r, q, t) \int_{-a}^{a}|f(v-u)| d u \\
& \quad+K_{2}(r, q, t) \int_{a}^{\infty} u^{-2}|f(v-u)| d u+K_{2}(r, q, t) \int_{-\infty}^{-a} u^{-2}|f(v-u)| d u<\infty
\end{aligned}
$$

for each $v \in R$ (the real line). It is known [11; Theorem 20] that if

$$
\frac{1}{2 T} \int_{-T}^{T}|g(v)|^{2} d v
$$

is bounded in $T$, then

$$
\int_{-\infty}^{\infty} \frac{|g(v)|^{2}}{v^{2}+1} d v<\infty
$$

Hence, to show that $P_{\lambda}(t) f$ is in $W^{2}$, it suffices to show that

$$
\frac{1}{2 T} \int_{-T}^{T}\left|\left(P_{\lambda}(t) f\right)(v)\right|^{2} d v
$$

is bounded in T. By the Schwartz inequality we see that

$$
\begin{aligned}
& \frac{1}{2 T} \int_{-T}^{T}\left|\left(P_{\lambda}(t) f\right)(v)\right|^{2} d v \leqq \frac{1}{2 T} \int_{-T}^{T}\left(\int_{-\infty}^{\infty}\left|p_{\lambda}(t, v-u) f(u)\right| d u\right)^{2} d v \\
& \leqq \frac{1}{2 T} \int_{-T}^{T}\left[\int_{-\infty}^{\infty}\left|p_{\lambda}(t, v-u)\right|^{2}\left(1+(v-u)^{2}\right) d u \int_{-\infty}^{\infty} \frac{|f(u)|^{2}}{(u-v)^{2}+1} d u\right] d v .
\end{aligned}
$$

From (1.1) it follows that

$$
\int_{-\infty}^{\infty}\left|p_{\lambda}(t, x)\right|^{2}\left(1+x^{2}\right) d x<\infty
$$

Also it is known [11; pp. 168-169] that

$$
\frac{1}{2 T} \int_{-T}^{T}\left[\int_{-\infty}^{\infty} \frac{|f(u)|^{2}}{(v-u)^{2}+1} d u\right] d v
$$

is bounded in T. From these facts we obtain that (1.2) is bounded in T. Q.E.D.

We have the following 
LeMma 1.2. Let $\lambda=r-i q(r>0)$ and $t>0$. If $f$ is in $L^{2}(R)$ then

$$
\int_{-\infty}^{\infty} p_{\lambda}(t, u) f(v-u) d u=(2 \pi)^{-1 / 2} \int_{-\infty}^{\infty}\left(U_{w} f\right)(u) \exp (i u v-\lambda t|u|) d u
$$

where we let

$$
\left(U_{w} f\right)(u)=(U f)(u)=\lim _{a \rightarrow \infty}(2 \pi)^{-1 / 2} \int_{-a}^{a} f(w) \exp (-i w u) d w .
$$

Here 1.i.m denotes limit in the mean with index 2. cf. [3; Theorem 21, p. 974]

Lемма 1.3. Let $\lambda=r-i q(r>0)$ and $t>0$. Let $\alpha$ be a positive number. Then

1) if $0<t r<\alpha$,

$$
\begin{aligned}
& \left(P_{\lambda}(t) f\right)(z)=(z-i \alpha)(2 \pi)^{-1 / 2} \int_{-\infty}^{0}\left(U f_{\lambda t}\right)(u) \exp (\lambda t+i z) u d u \\
& \quad+(z-i \alpha)(2 \pi)^{-1 / 2} \int_{0}^{\infty}\left(U k_{\lambda t}\right)(u) \exp (-\lambda t+i z) u d u \\
& \quad+\left(\frac{2}{\pi}\right)^{1 / 2} i \lambda t \int_{0}^{\infty}\left(U k_{\lambda t}\right)(u) \exp (\lambda t-\alpha) u d u
\end{aligned}
$$

2) if $\alpha<t r$,

$$
\begin{aligned}
& \left(P_{\lambda}(t) f\right)(z)=(z-i \alpha)(2 \pi)^{-1 / 2} \int_{-\infty}^{0}\left(U f_{\lambda t}\right)(u) \exp (\lambda t+i z) u d u \\
& \quad+(z-i \alpha)(2 \pi)^{-1 / 2} \int_{0}^{\infty}\left(U k_{\lambda t}\right)(u) \exp (-\lambda t+i z) u d u \\
& \quad-\left(\frac{2}{\pi}\right)^{1 / 2} i \lambda t \int_{-\infty}^{0}\left(U k_{\lambda t}\right)(u) \exp (\lambda t-\alpha) u d u,
\end{aligned}
$$

where we let

$$
f_{\lambda t}(u)=\frac{f(u)}{u-i \alpha+i \lambda t}, k_{\lambda s}(u)=\frac{f(u)}{u-i \alpha-i \lambda t} .
$$

Proof. It is easy to show that

$$
\begin{aligned}
p_{\lambda}(t, u-z)=\frac{1}{2 \pi} & {\left[\frac{z-i \alpha}{(u-i \alpha+i \lambda t)(\lambda t-i(u-z))}+\frac{z-i \alpha}{(u-i \alpha-i \lambda t)(\lambda t+i(u-z))}\right.} \\
& \left.+\frac{2 \lambda t}{(u-i \alpha-i \lambda t)(u-i \alpha+i \lambda t)}\right] .
\end{aligned}
$$

Hence we have

$$
\begin{aligned}
\left(P_{\lambda}(t) f\right)(v)= & (z-i \alpha) \frac{1}{2 \pi} \int_{-\infty}^{\infty} \frac{f(u) d u}{(u-i \alpha+i \lambda t)(\lambda t+i(z-u))} \\
& +(z-i \alpha) \frac{1}{2 \pi} \int_{-\infty}^{\infty} \frac{f(u) d u}{(u-i \alpha-i \lambda t)(\lambda t-i(z-u))}
\end{aligned}
$$




$$
+\frac{\lambda t}{\pi} \int_{-\infty}^{\infty} \frac{f(u) d u}{(u-i \alpha-i \lambda t)(u-i \alpha+i \lambda t)}
$$

By the Parseval theorem it holds that

$$
\begin{gathered}
\int_{-\infty}^{\infty} \frac{f(u) d u}{(u-i \alpha+i \lambda t)(\lambda t+i(z-u))}=(2 \pi)^{1 / 2} \int_{-\infty}^{0}\left(U f_{\lambda t}\right)(u) \exp (\lambda t u+i z u) d u \\
\int_{-\infty}^{\infty} \frac{f(u) d u}{(u-i \alpha-i \lambda t)(\lambda t-i(z-u))}=(2 \pi)^{1 / 2} \int_{0}^{\infty}\left(U k_{\lambda t}\right)(u) \exp (-\lambda t u+i z u) d u \\
\int_{-\infty}^{\infty} \frac{f(u) d u}{(u-i \alpha-i \lambda t)(u-i \alpha+i \lambda t)} \\
=\left\{\begin{array}{l}
(2 \pi)^{1 / 2} i \int_{0}^{\infty}\left(U k_{\lambda t}\right)(u) \exp (\lambda t-\alpha) u d u \text { if } 0<t r<\alpha \\
-(2 \pi)^{1 / 2} i \int_{-\infty}^{0}\left(U k_{\lambda t}\right)(u) \exp (\lambda t-\alpha) u d u \text { if } \alpha<t r
\end{array}\right.
\end{gathered}
$$

(cf. [1, p. 128], [11, Theorem 3]). From these equalities and from (1.4) we obtain (1.3) and (1.3').

Q.E.D.

LEMMA 1.4. Let $\lambda=r-i q(r>0)$ and $t>0$. Then $P_{\lambda}(t)$ is a linear bounded operator on $W^{2}$.

Proof. It is clear that $P_{\lambda}(t)$ is linear. Let $\alpha$ be a positive number such that $1 \leqq \alpha$ and $0<t r<\alpha$. By the Minkowski inequality and by Plancherel's theorem, and by the Schwartz inequality we see that

$$
\begin{aligned}
& \left\|P_{\lambda}(t) f\right\| \leqq\left[\int_{-\infty}^{\infty} \frac{z^{2}+\alpha^{2}}{z^{2}+1}\left|(2 \pi)^{-1 / 2} \int_{-\infty}^{0}\left(U f_{\lambda t}\right)(u) \exp (\lambda t+i z) u d u\right|^{2} d z\right]^{1 / 2} \\
& +\left[\int_{-\infty}^{\infty} \frac{z^{2}+\alpha^{2}}{z^{2}+1}\left|(2 \pi)^{-1 / 2} \int_{0}^{\infty}\left(U k_{\lambda t}\right)(u) \exp (-\lambda t+i z) u d u\right|^{2} d z\right]^{1 / 2} \\
& +\left[\int_{-\infty}^{\infty}-\frac{1}{z^{2}+I}\left|\left(\frac{2}{\pi}\right)^{1 / 2} i \lambda t \int_{0}^{\infty}\left(U k_{\lambda t}\right)(u) \exp (\lambda t-\alpha) u d u\right|^{2} d z\right]^{1 / 2} \\
& \leqq \alpha\left[\int_{-\infty}^{0}\left|\left(U f_{\lambda t}\right)(u)\right|^{2} d u\right]^{1 / 2}+\alpha\left[\int_{0}^{\infty}\left|\left(U k_{\lambda t}\right)(u)\right|^{2} d u\right]^{1 / 2} \\
& +\left(\frac{2}{\pi}\right)^{1 / 2}|\lambda| t\left[\int_{0}^{\infty}\left|\left(U k_{\lambda t}\right)(u)\right|^{2} d u\right]^{1 / 2} \\
& \cdot\left[\int_{0}^{\infty}|\exp (\lambda t-\alpha) u|^{2} d u\right]^{1 / 2}\left[\int_{-\infty}^{\infty} \frac{d z}{z^{2}+1}\right]^{1 / 2} \\
& \leqq\left[\alpha \sup _{u \in R}\left|\frac{u-i}{u-i \alpha+i \lambda t}\right|+\alpha \sup _{u \in R}\left|\frac{u-i}{u-i \alpha-i \lambda t}\right|\right]\|f\| \\
& +\left(\frac{2}{\pi}\right)^{1 / 2}|\lambda| t\left[\sup _{u \in R}\left|\frac{u-i}{u-i \alpha-i \lambda t}\right|\right] \pi^{1 / 2}\left[-\frac{1}{2(t r-\alpha)}\right]^{1 / 2}\|f\| .
\end{aligned}
$$


Since

$$
\sup _{u \in R}\left|\frac{u-i}{u-i \alpha+i \lambda t}\right|, \sup _{u \in R}\left|\frac{u-i}{u-i \alpha-i \lambda t}\right|
$$

are bounded, from (1.5) it follows that $P_{\lambda}(t)$ is a bounded operator on $W^{2}$.

Q.E.D.

Theorem 1.1. Let $\lambda=r-i q(r>0)$. Let $P_{\lambda}(0)=I$, where $I$ denotes the identity operator on $W^{2}$. Then a family $\left\{P_{\lambda}(t): 0 \leqq t<\infty\right\}$ of linear bounded operators on $W^{2}$ is a semigroup of class $\left(C_{0}\right)$.

Proof. Semigroup property $P_{\lambda}(t) P_{\lambda}(s)=P_{\lambda}(t+s)$ : Let $0<s, t<\infty$. First let $f$ be a continuous function with compact support. Then we have

$$
\begin{aligned}
\left(P_{\lambda}(t) P_{\lambda}(s) f\right)(v) & =\int_{-\infty}^{\infty} p_{\lambda}(t, u) d u \int_{-\infty}^{\infty} p_{\lambda}(s, v-u-y) f(y) d y \\
& =\int_{-\infty}^{\infty} f(y) d y \int_{-\infty}^{\infty} p_{\lambda}(t, u) p_{\lambda}(s, v-u-y) d u \\
& =\int_{-\infty}^{\infty} p_{\lambda}(t+s, v-y) f(y) d y=\left(P_{\lambda}(t+s) f\right)(v)
\end{aligned}
$$

(cf. [8, proof of Lemma 1.3]). When $f$ is in $W^{2}$, taking a sequence of continuous functions $f_{n}$ with compact support converging to $f$ in the norm we obtain

$$
\begin{aligned}
\left\|P_{\lambda}(t) P_{\lambda}(s) f-P_{\lambda}(t+s) f\right\| \leqq & \left\|P_{\lambda}(t) P_{\lambda}(s) f-P_{\lambda}(t) P_{\lambda}(s) f_{n}\right\| \\
& +\left\|P_{\lambda}(t+s) f_{n}-P_{\lambda}(t+s) f\right\| .
\end{aligned}
$$

By Lemma 1.4

$$
\begin{aligned}
& \left\|P_{\lambda}(t) P_{\lambda}(s)\left(f-f_{n}\right)\right\| \leqq\left\|P_{\lambda}(t)\right\|\left\|P_{\lambda}(s)\right\|\left\|f-f_{n}\right\| \longrightarrow 0 \text { and } \\
& \left\|P_{\lambda}(t+s)\left(f-f_{n}\right)\right\| \leqq\left\|P_{\lambda}(t+s)\right\|\left\|f-f_{n}\right\| \longrightarrow 0
\end{aligned}
$$

as $n \rightarrow \infty$, hence $P_{\lambda}(t) P_{\lambda}(s) f=P_{\lambda}(t+s) f$ for $f$ in $W^{2}$.

Strong continuity $P_{\lambda}(s) f \rightarrow P_{\lambda}(t) f(s \rightarrow t)$ : By the semigroup property it suffices to prove that

(a) $P_{\lambda}(t) f \rightarrow f$ as $t \rightarrow+0$ in the norm for all $f$ in $W^{2}$.

It is known [4, Theorem 10.6.5] that (a) is equivalent to

(b) $\left(P_{\lambda}(t) f, g\right) \rightarrow(f, g)$ as $t \rightarrow+0$ for all $f, g$ in $W^{2}$.

Let us prove (b). First let $f$ be a continuous function with compact support. Then by Lemma 1.2 and by the Parseval theorem we obtain

$$
\begin{aligned}
\left(P_{\dot{a}}(t) f, g\right) & =\int_{-\infty}^{\infty}\left[\int_{-\infty}^{\infty}(U f)(u) \exp (i v u-\lambda t|u|) d u\right] \overline{\frac{g(v)}{v^{2}+1}} d v \\
& \left.\left.=\int_{-\infty}^{\infty}(U f)(u) \exp (-\lambda t|u|) \overline{U_{v}\left[g ( v ) \left(v^{2}+1\right.\right.}\right)^{-1}\right] \overline{(u)} d u
\end{aligned}
$$




$$
\longrightarrow \int_{-\infty}^{\infty}(U f)(u) \overline{U_{v}\left[g(v)\left(v^{2}+1\right)^{-1}\right](u)} d u \quad \text { as } \quad t \longrightarrow+0 .
$$

By the Parseval theorem

$$
\int_{-\infty}^{\infty}(U f)(u) \overline{U_{v}\left[g(v)\left(v^{2}+1\right)^{-1}\right](u)} d u=\int_{-\infty}^{\infty} f(v) \frac{\overline{g(v)}}{v^{2}+1} d v,
$$

hence it follows that $\left(P_{\lambda}(t) f, g\right) \rightarrow(f, g)$ as $t \rightarrow+0$ for the above function $f$. If $f$ is in $W^{2}$, taking a sequence of continuous functions $f_{n}$ with compact support converging to $f$ in the norm we have

$$
\begin{aligned}
\left|\left(P_{\lambda}(t) f, g\right)-(f, g)\right| & \leqq\left|\left(P_{\lambda}(t)\left(f-f_{n}\right), g\right)\right| \\
& +\left|\left(P_{\lambda}(t) f_{n}, g\right)-\left(f_{n}, g\right)\right|+\left|\left(f_{n}, g\right)-(f, g)\right| .
\end{aligned}
$$

It is seen from (1.5) that $P_{\lambda}(t)$ is uniformly bounded in the neighborhood at $t=0$. Hence from (1.6) it follows that

$$
\left|\left(P_{\lambda}(t) f, g\right)-(f, g)\right| \longrightarrow 0 \text { as } t \longrightarrow+0 .
$$

We have a following

Remark 1.1. Let $f$ be in $W^{2}$. Then $P_{\lambda}(t) f$ is strongly continuous in $\lambda$ on $C^{+}=\{\lambda:$ Real part of $\lambda$ is positive $\}$.

LEMMA 1.5. Let $q \neq 0$ be given and let $f$ be in $W^{2}$. Let

$$
\begin{aligned}
& \left(P_{-i q}(t) f\right)(z)=(z-i \alpha) \underset{a \rightarrow \infty}{\lim }(2 \pi)^{-1 / 2} \int_{-a}^{0}\left(U f_{-i q t}\right)(u) \exp (-i q t+i z) u d u \\
& +(z-i \alpha) \operatorname{li}_{a \rightarrow \infty}(2 \pi)^{-1 / 2} \int_{0}^{a}\left(U k_{-i q t}\right)(u) \exp (i q t+i z) u d u \\
& +\left(\frac{2}{\pi}\right)^{1 / 2} q t \int_{0}^{\infty}\left(U k_{-q t}\right)(u) \exp (i q t-\alpha) u d u
\end{aligned}
$$

for a positive number $\alpha \geqq 1$ and $t>0$. Then $P_{r-i q}(t) f \rightarrow P_{-i q}(t) f$ as $r \rightarrow+0$ in the norm and $P_{-i q}(t)$ is a linear bounded operator on $W^{2}$.

Proof. Let $\lambda=r-i q(r>0)$. Suppose $0<t r<1 \leqq \alpha$. By $(1.3)$ and by the Minkowski inequality, and by Plancherel's theorem we see that

$$
\begin{aligned}
& \left\|P_{\lambda}(t) f-P_{-i q}(t) f\right\| \leqq\left[\int_{-\infty}^{\infty} \frac{z^{2}+\alpha^{2}}{z^{2}+1} \mid(2 \pi)^{-1 / 2} \int_{-\infty}^{0}\left(U f_{\lambda t}\right)(u) \exp (\lambda t+i z) u d u\right. \\
& \left.\quad-\left.\underset{a \rightarrow \infty}{\lim (2 \pi)^{-1 / 2}} \int_{-a}^{0}\left(U f_{-i q t}\right)(u) \exp (-i q t+i z) u d u\right|^{2} d z\right]^{1 / 2} \\
& +\left[\int_{-\infty}^{\infty} \frac{z^{2}+\alpha^{2}}{z^{2}+1} \mid(2 \pi)^{-1 / 2} \int_{0}^{\infty}\left(U k_{\lambda t}\right)(u) \exp (-\lambda t+i z) u d u\right. \\
& \left.-\left.\underset{a \rightarrow \infty}{\lim (2 \pi)^{-1 / 2}} \int_{0}^{a}\left(U k_{-i q t}\right)(u) \exp (i q t+i z) u d u\right|^{2} d z\right]^{1 / 2}
\end{aligned}
$$




$$
\begin{aligned}
& +\left[\int_{-\infty}^{\infty} \frac{1}{z^{2}+1} \mid\left(\frac{2}{\pi}\right)^{1 / 2} i \lambda t \int_{0}^{\infty}\left(U k_{\lambda t}\right)(u) \exp (\lambda t-\alpha) u d u\right. \\
& \left.-\left.\left(\frac{2}{\pi}\right)^{1 / 2} q t \int_{0}^{\infty}\left(U k_{-i q t}\right)(u) \exp (-i q t-\alpha) u d u\right|^{2} d z\right]^{1 / 2} \\
& \leqq \alpha\left[\int_{-\infty}^{0} \mid\left(U f_{\lambda t}\right)(u) e^{\lambda t u}-\left(U f_{-i q t}\right)(u) e^{-\left.i q t u\right|^{2}} d u\right]^{1 / 2} \\
& +\alpha\left[\int_{0}^{\infty}\left|\left(U k_{\lambda t}\right)(u) e^{-\lambda t u}-\left(U k_{-i q t}\right)(u) e^{i q t u}\right|^{2} d u\right]^{1 / 2} \\
& +2^{1 / 2} t\left|\int_{0}^{\infty} \lambda\left(U k_{\lambda t}\right)(u) e^{(\lambda t-\alpha) u} d u-\int_{0}^{\infty} q\left(U k_{-i q t}\right)(u) e^{(-i q t-\alpha) u} d u\right| \\
& \longrightarrow 0 \text { as } r \longrightarrow+0 .
\end{aligned}
$$

In the same manner as the proof of Lemma 1.4 we can prove that $P_{-i q}(t)$ is a bounded operator on $W^{2}$.

Q.E.D.

REMARK 1.2. If $f$ is in $L^{2}(R)$,

$$
\left(P_{-i q}(t) f\right)(v)=\underset{a \rightarrow \infty}{\lim }(2 \pi)^{-1 / 2} \int_{-a}^{a}(U f)(u) \exp (i u v+i q t|u|) d u .
$$

This follows from Lemma 1.2.

TheOREM 1.2. Let $q \neq 0$ be given and let $\alpha$ be the same number as in Lemma 1.5. When $t>0$, let

$$
\begin{aligned}
& \left(P_{-i q}(-t) f\right)(z)=(z-i \alpha) \underset{a \rightarrow \infty}{\lim }(2 \pi)^{-1 / 2} \int_{-a}^{0}\left(U f_{i q q}\right)(u) \exp (i q t+i z) u d u \\
& +(z-i \alpha) \lim _{a \rightarrow \infty}(2 \pi)^{-1 / 2} \int_{0}^{a}\left(U k_{i q q}\right)(u) \exp (-i q t+i z) u d u \\
& \quad-\left(\frac{2}{\pi}\right)^{1 / 2} q t \int_{0}^{\infty}\left(U k_{i q q}\right)(u) \exp (i q t-\alpha) u d u
\end{aligned}
$$

for $f$ in $W^{2}$. Then $P_{-i q}(-t)$ is a linear bounded operator on $W^{2}$ and

$$
P_{-i q}(t) P_{-i q}(-t)=P_{-i q}(-t) P_{-i q}(t)=I .
$$

A family $\left\{P_{-i q}(t):-\infty<t<\infty\right\}$ is a strongly continuous group of linear operators on $W^{2}$, where let $P_{-i q}(0)=I$.

Proof. In the same manner as the proof of Lemma 1.4 we can prove that $P_{-i q}(-t)$ is a linear bounded operator on $W^{2}$. Let us prove (1.9). Let $S(R)$ be the space of rapidly decreasing and infinitely differentiable functions. It is easy to show that $S(R)$ is dense in $W^{2}$. First let us prove that

$$
\left(P_{-i q}(-t) f\right)(z)=(2 \pi)^{-1 / 2} \int_{-\infty}^{\infty}(U f)(u) \exp (i z u-i q t|u|) d u
$$


for all $f$ in $S(R)$. Let $f$ be in $S(R)$. By the Parseval theorem we see that

$$
\begin{aligned}
\left(U f_{i q t}\right)(u) & =(2 \pi)^{-1 / 2} \int_{-\infty}^{\infty} \frac{f(y)}{y-t q-i \alpha} \exp (-i y u) d y \\
& =(2 \pi)^{-1 / 2} \int_{-\infty}^{\infty}(U f)(y) U_{k \cdot[}\left[\frac{1}{w-t q-i \alpha}\right](u-y) d y \\
& =i \int_{u}^{\infty}(U f)(y) \exp (i t q-\alpha) y d y \exp (\alpha-i t q) u
\end{aligned}
$$

(cf. $[1$, p. 128], [11, Theorem 3]). Hence we obtain by the Fubini theorem that (the first term of $(1.8))=(z-i \alpha)(2 \pi)^{-1 / 2} \int_{-\infty}^{0}\left[i \int_{u}^{\infty}(U f)(y) \exp (i t q-\alpha) y d y\right]$ $\cdot \exp (\alpha+i z) u d u$

$$
\begin{aligned}
= & (z-i \alpha)(2 \pi)^{-1 / 2} i\left[\int_{-\infty}^{0}(U f)(y) \exp (i t q-\alpha) y d y \int_{-\infty}^{y} \exp (\alpha+i z) u d u\right. \\
& \left.+\int_{0}^{\infty}(U f)(y) \exp (i t q-\alpha) y d y \int_{-\infty}^{0} \exp (\alpha+i z) u d u\right] \\
= & (2 \pi)^{-1 / 2}\left[\int_{-\infty}^{0}(U f)(y) \exp (i t q+i z) y d y+\int_{0}^{\infty}(U f)(y) \exp (i t q-\alpha) y d y\right] .
\end{aligned}
$$

Also by the Parseval theorem we see that

$$
\begin{aligned}
\left(U k_{i q t}\right)(u) & =(2 \pi)^{-1 / 2} \int_{-\infty}^{\infty} \frac{f(y)}{y+t q-i \alpha} \exp (-i y u) d y \\
& =(2 \pi)^{-1 / 2} \int_{-\infty}^{\infty}(U f)(y) U_{w}\left[\frac{1}{w+t q-i \alpha}\right](u-y) d y \\
& =i \int_{u}^{\infty}(U f)(y) \exp (-i t q-\alpha) y d y \exp (i t q+\alpha) u
\end{aligned}
$$

Hence we obtain that

(the second term of (1.8))

$$
=(z-i \alpha)(2 \pi)^{-1 / 2} \int_{0}^{\infty}\left[i \int_{u}^{\infty}(U f)(y) \exp (-i t q-\alpha) y d y\right] \exp (\alpha+i z) u d u
$$

(by the Funini theorem)

$$
\begin{array}{r}
=(z-i \alpha)(2 \pi)^{-1 / 2} i \int_{0}^{\infty}(U f)(y) \exp (-i t q-\alpha) y d y \int_{0}^{y} \exp (\alpha+i z) u d u \\
=(2 \pi)^{-1 / 2} \int_{0}^{\infty}(U f)(y) \exp (-i t q+i z) y d y-(2 \pi)^{-1 / 2} \int_{0}^{\infty}(U f)(y) \\
\cdot \exp (-\alpha-i t q) y d y .
\end{array}
$$

By the Parseval theorem we see that 
(the third term of (1.8))

$$
\begin{aligned}
& =(2 \pi)^{-1} 2 i q t \int_{-\infty}^{\infty} \frac{f(v) d v}{(v+t q-i \alpha)(v-t q-i \alpha)} \\
& =-(2 \pi)^{-1} i\left[\int_{-\infty}^{\infty} \frac{f(v) d v}{v+t q-i \alpha}-\int_{-\infty}^{\infty} \frac{f(v) d v}{v-t q-i a}\right] \\
& =(2 \pi)^{-1 / 2} \int_{0}^{\infty}(U f)(u)(\exp (-i t q-\alpha) u-\exp (i t q-\alpha) u) d u .
\end{aligned}
$$

From (1.11), (1.12) and (1.13) we obtain (1.10). By (1.10) and by the fact that $P_{-i q}(-t)$ is a bounded operator, it holds that

$$
\left(P_{-i q}(-t) f\right)(z)=\underset{a \rightarrow \infty}{\lim }(2 \pi)^{-1 / 2} \int_{-a}^{a}(U f)(u) \exp (i z u-i q t|u|) d u
$$

for $f$ in $L^{2}(R)$. Hence if $f$ is in $L^{2}(R)$, from Remark 1.2 and (1.14) it follows that

$$
P_{-i q}(t) P_{-i q}(-t) f=P_{-i q}(-t) P_{-i q}(t) f=f
$$

By this equality and by the fact that $P_{-i q}(t)$ and $P_{-i q}(-t)$ are bounded operators, we obtain (1.15) for all $f$ in $W^{2}$.

From Remark 1.2, (1.14) and from the fact that $P_{-i q}(t), P_{-i q}(-t)$ are bounded operators it follows that

$$
P_{-i q}(t) P_{-i q}(s)=P_{-i q}(t+s)
$$

for $-\infty<t, s<\infty$. In the same manner as the proof of Theorem 1.1 we can show that $\left\{P_{-i q}(t): 0 \leqq t<\infty\right\}$ is a semigroup of class $\left(C_{0}\right)$. Hence by this fact and by (1.16) the family $\left\{P_{-i q}(t):-\infty<t<\infty\right\}$ is a one-parameter strongly continuous group.

Q.E.D.

2. Infinitesimal generators and its domains. In what follows, for simplicity let $n(u)=f(u) /(u-i)$ for $f$ in $W^{2}$. In the following discussions, suppose that $\alpha=1$ in Lemma 1.3, Lemma 1.5 and Theorem 1.2.

Theorem 2.1. The infinitesimal generator $A_{\lambda}$ of the semigroup $\left\{P_{\lambda}(t)\right.$ : $0 \leqq t<\infty$ \} in Theorem 1.1 is given by the following form;

$$
\begin{aligned}
D\left(A_{\lambda}\right)= & \left\{f \in W^{2}:\left\{u \mid n(u) \in L^{2}(R)\right\} \text { and for } f \text { in } D\left(A_{\lambda}\right)\right. \\
\left(A_{\lambda} f\right)(z)= & i \lambda \operatorname{li.m}_{a \rightarrow \infty}(2 \pi)^{-1 / 2} \int_{-a}^{a}(U n)(u)(\operatorname{sign} u) \exp (i z u) d u \\
& -\lambda(z-i) \underset{a \rightarrow \infty}{\operatorname{lim.m}}(2 \pi)^{-1 / 2} \int_{-a}^{a}|u|(U n)(u) \exp (i z u) d u
\end{aligned}
$$

Proof. Let $D\left(A_{\lambda}\right)$ denote the domain of the infinitesimal generator $A_{\lambda}$. When $f$ is in $D\left(A_{\lambda}\right)$, let $A_{\lambda} f=g$. Let us put 


$$
H(\lambda t, u)= \begin{cases}\left(U f_{\lambda s}\right)(u) e^{\lambda t u} & \text { for } u \leqq 0 \\ \left(U k_{\lambda s}\right)(u) e^{-\lambda t u} & \text { for } u>0\end{cases}
$$

and

$$
L(\lambda t, z)=\left(\frac{2}{\pi}\right)^{1 / 2} i \lambda \int_{0}^{\infty}\left(U k_{\lambda t}\right)(u) \exp (\lambda t-1) u d u \frac{1}{z-i} .
$$

Note that

$$
f(z)=(z-i) \lim _{a \rightarrow \infty}(2 \pi)^{-1 / 2} \int_{-a}^{a}(U n)(u) \exp (i z u) d u .
$$

Then by definition of the infinitesimal generator $A_{\lambda}$ we have

$$
\begin{aligned}
& \left\|t^{-1}\left(P_{\lambda}(t) f-f\right)-A_{\lambda} f\right\| \\
& =\left[\int_{-\infty}^{\infty} \mid t^{-1}\left\{(2 \pi)^{-1 / 2} \int_{-\infty}^{\infty} H(\lambda t, u) \exp (i z u) d u\right.\right. \\
& \left.\quad-\operatorname{lin}_{a \rightarrow \infty}(2 \pi)^{-1 / 2} \int_{-a}^{a}(U n)(u) \exp (i z u) d u\right\} \\
& \left.\quad+L(\lambda t, z)-\left.\frac{g(z)}{z-i}\right|^{2} d z\right]^{1 / 2} \longrightarrow 0 \text { as } t \longrightarrow+0
\end{aligned}
$$

Let us put

$$
K(z)=\left(\frac{2}{\pi}\right)^{1 / 2} q \int_{0}^{\infty}(U n)(u) \exp (-u) d u \frac{1}{z-i} .
$$

It is easy to show that $L(\lambda t, z) \rightarrow K(z)$ as $t \rightarrow+0$ in the $L^{2}$-norm. Hence, if we let

$$
g_{1}(z)=\frac{g(z)}{z-i}, g_{2}(z)=g_{1}(z)-K(z),
$$

then $g_{2}(z) \in L^{2}(R)$, and by Plancherel's theorem and by (2.2) we see that

$$
\begin{aligned}
& {\left[\int_{-\infty}^{\infty} \mid t^{-1}\left\{(2 \pi)^{-1 / 2} \int_{-\infty}^{\infty} H(\lambda t, u) \exp (i z u) d u\right.\right.} \\
& \left.\left.\quad-\lim _{a \rightarrow \infty}(2 \pi)^{-1 / 2} \int_{-a}^{a}(U n)(u) \exp (i z u) d u\right\}-\left.g_{2}(z)\right|^{2} d z\right]^{1 / 2} \\
& =\left[\int_{-\infty}^{\infty}\left|(H(\lambda t, u)-(U n)(u)) t^{-1}-\left(U g_{2}\right)(u)\right|^{2} d u\right]^{1 / 2} \\
& =\left[\int_{0}^{\infty}\left|\left\{\left(U k_{\lambda s}\right)(u) \exp (-\lambda t u)-(U n)(u)\right\} t^{-1}-\left(U g_{2}\right)(u)\right|^{2} d u\right. \\
& \left.+\int_{-\infty}^{0}\left|\left\{\left(U f_{\lambda t}\right)(u) \exp (\lambda t u)-(U n)(u)\right\} t^{-1}-\left(U g_{2}\right)(u)\right|^{2} d u\right]^{1 / 2} \\
& \longrightarrow 0 \text { as } t \longrightarrow+0 .
\end{aligned}
$$


Hence

$$
\begin{aligned}
& {\left[\int_{0}^{\infty} \mid\left\{\left(U k_{\lambda t}\right)(u) \exp (-\lambda t u)-(U n)(u)\right\} t^{-1}\right.} \\
& \left.-\left.\left(U g_{2}\right)(u)\right|^{2} d u\right]^{1 / 2} \longrightarrow 0 \text { as } t \longrightarrow+0 \\
& {\left[\int_{-\infty}^{0} \mid\left\{\left(U f_{\lambda t}\right)(u) \exp (\lambda t u)-(U n)(u)\right\} t^{-1}\right.} \\
& \left.-\left.\left(U g_{2}\right)(u)\right|^{2} d u\right]^{1 / 2} \longrightarrow 0 \text { as } t \longrightarrow+0
\end{aligned}
$$

Let us show that (2.3) and (2.4) hold if and only if $|u|(U n)(u)$ is $L^{2}$-integrable.

Suppose that (2.3) and (2.4) hold. We see that

$$
\begin{aligned}
& \int_{0}^{\infty}\left|\left[\left(U k_{\lambda t}\right)(u) \exp (-\lambda t u)-(U n)(u)\right] t^{-1}-\left(U g_{2}\right)(u)\right|^{2} d u \\
= & \int_{0}^{\infty}\left|\left[\left(U k_{\lambda t}-U n\right)(u) \exp (-\lambda t u)+(\exp (-\lambda t u)-1)(U n)(u)\right] t^{-1}-\left(U g_{2}\right)(u)\right|^{2} d u \\
= & \int_{0}^{\infty}\left|\exp (-\lambda t u) U\left(t^{-1}\left(k_{\lambda t}-n\right)\right)(u)+t^{-1}(\exp (-\lambda t u)-1)(U n)(u)-\left(U g_{2}\right)(u)\right|^{2} d u .
\end{aligned}
$$

Also

$$
\begin{array}{r}
\int_{-\infty}^{0}\left|\left[\left(U f_{\lambda \varepsilon}\right)(u) \exp (\lambda t u)-(U n)(u)\right] t^{-1}-\left(U g_{2}\right)(u)\right|^{2} d u \\
=\int_{-\infty}^{0} \mid \exp (\lambda t u) U\left(t^{-1}\left(f_{\lambda s}-n\right)\right)(u)+t^{-1}(\exp (\lambda t u)-1)(U n)(u) \\
-\left.\left(U g_{2}\right)(u)\right|^{2} d u .
\end{array}
$$

It is seen that for some function $Q(u)$ in $L^{2}([0, \infty))$

$$
\int_{0}^{\infty}\left|\exp (-\lambda t u) U\left(t^{-i}\left(k_{\lambda t}-n\right)\right)(u)-Q(u)\right|^{2} d u \longrightarrow 0 \text { as } t \longrightarrow+0 .
$$

Hence by (2.3) and (2.5) we see that for some function $R_{1}(u)=\left(U g_{2}\right)(u)-Q(u)$ in $L^{2}([0, \infty))$

$$
\int_{0}^{\infty}\left|t^{-1}(\exp (-\lambda t u)-1)(U n)(u)-R_{1}(u)\right|^{2} d u \longrightarrow 0 \text { as } t \longrightarrow+0 .
$$

On the otherhand, clearly

$$
t^{-1}(\exp (-\lambda t u)-1)(U n)(u) \longrightarrow-\lambda u(U n)(u) \text { as } t \longrightarrow+0
$$

for almost all $u \in[0, \infty)$. By (2.7) and (2.8) we obtain that $R_{1}(u)=-\lambda u(U n)(u)$ for almost all $u \in[0, \infty)$. In the same manner, from (2.4) and (2.6) we see that for some function $R_{2}(u)$ in $L^{2}((-\infty, 0])$ 


$$
\int_{-\infty}^{0}\left|t^{-1}(\exp (\lambda t u)-1)(U n)(u)-R_{2}(u)\right|^{2} d u \longrightarrow 0 \text { as } t \longrightarrow+0
$$

and $R_{2}(u)=\lambda u(U n)(u)$ for almost all $u \in(-\infty, 0]$. Hence if (2.3) and (2.4) hold, $|u|(U n)(u)$ is in $L^{2}(R)$.

Conversely if $f$ is a function in $W^{2}$ such that $|u|(U n)(u) \in L^{2}(R)$, from the above argument it is clear that $f$ is in $D\left(A_{\lambda}\right)$. Lastly we obtain

$$
D\left(A_{\lambda}\right)=\left\{f \in W^{2}:|u|(U n)(u) \in L^{2}(R)\right\} .
$$

Next let us show that if $f$ is in $D\left(A_{\lambda}\right), A_{\lambda} f$ is given by (2.1). Let $B_{\lambda} f$ be the right hand side of (2.1). By the Minkowski inequality we see that

$$
\begin{aligned}
& \left\|t^{-1}\left(P_{\lambda}(t) f-f\right)-B_{\lambda} f\right\| \\
& \leqq\left[\int_{-\infty}^{\infty} \mid\left[( z - i ) \left\{(2 \pi)^{-1 / 2} \int_{-\infty}^{0}\left(U f_{\lambda z}\right)(u) \exp (\lambda t u+i z u) d u\right.\right.\right. \\
& \left.-\lim _{a \rightarrow \infty}(2 \pi)^{-1 / 2} \int_{-a}^{0}(U n)(u) \exp (i z u) d u\right\} t^{-1} \\
& +i \lambda \lim _{a \rightarrow \infty}(2 \pi)^{-1 / 2} \int_{-a}^{0}(U n)(u) \exp (i z u) d u \\
& +\lambda(z-i) \operatorname{lit.m}_{a \rightarrow \infty}(2 \pi)^{-1 / 2} \int_{-a}^{0}|u|(U n)(u) \exp (i z u) d u \\
& \left.\left.+i \lambda(2 \pi)^{-1 / 2} \int_{0}^{\infty}(U n)(u) \exp (-u) d u\right]\left.\right|^{2} \frac{d z}{z^{2}+1}\right]^{1 / 2} \\
& +\left[\int_{-\infty}^{\infty} \mid\left[( z - i ) \left\{(2 \pi)^{-1 / 2} \int_{0}^{\infty}\left(U k_{\lambda t}\right)(u) \exp (-\lambda t u+i z u) d u\right.\right.\right. \\
& \left.-\lim _{a \rightarrow \infty}(2 \pi)^{-1 / 2} \int_{0}^{a}(U n)(u) \exp (i z u) d u\right\} t^{-1} \\
& -i \lambda \lim _{a \rightarrow \infty}(2 \pi)^{-1 / 2} \int_{0}^{a}(U n)(u) \exp (i z u) d u \\
& +\lambda(z-i) \lim _{a \rightarrow \infty} \int_{0}^{a}|u|(U n)(u) \exp (i z u) d u \\
& \left.\left.+i \lambda(2 \pi)^{-1 / 2} \int_{0}^{\infty}(U n)(u) \exp (-u) d u\right]\left.\right|^{2} \frac{d z}{z^{2}+1}\right]^{1 / 2} \\
& +\left[\int_{-\infty}^{\infty} \mid\left(\frac{2}{\pi}\right)^{1 / 2} i \lambda\left\{\int_{0}^{\infty}\left(U k_{\lambda t}\right)(u) \exp (\lambda t-1) u d u\right.\right. \\
& \left.\left.-\int_{0}^{\infty}(U n)(u) \exp (-u) d u\right\}\left.\right|^{2} \frac{d z}{z^{2}+1}\right]^{1 / 2} \text {. }
\end{aligned}
$$

By the Minkowski inequality we have

(the first term of (2.9)) 


$$
\begin{aligned}
& \leqq\left[\int_{-\infty}^{\infty} \mid(2 \pi)^{-1 / 2} \int_{-\infty}^{0}\left(\left(U f_{\lambda t}\right)(u)-(U n)(u)\right) t^{-1} \exp (\lambda t u+i z u) d u\right. \\
& +\frac{i \lambda}{z-i} \operatorname{lif.m}_{a \rightarrow \infty}(2 \pi)^{-1 / 2} \int_{-a}^{0}(U n)(u) \exp (i z u) d u \\
& \left.+\left.\frac{i \lambda}{z-i}(2 \pi)^{-1 / 2} \int_{0}^{\infty}(U n)(u) \exp (-u) d u\right|^{2} d z\right]^{1 / 2} \\
& +\left[\int_{-\infty}^{\infty} \mid\left\{(2 \pi)^{-1 / 2} \int_{-\infty}^{0}(U n)(u) \exp (\lambda t u+i z u) d u\right.\right. \\
& \left.-\lim _{a \rightarrow \infty}(2 \pi)^{-1 / 2} \int_{-a}^{0}(U n)(u) \exp (i z u) d u\right\} t^{-1} \\
& \left.+\left.\lambda \lim _{a \rightarrow \infty}(2 \pi)^{-1 / 2} \int_{-a}^{0}|u|(U n)(u) \exp (i z u) d u\right|^{2} d z\right]^{1 / 2} .
\end{aligned}
$$

By the Parseval theorem it holds that

$$
\begin{aligned}
& {\left[\left(U f_{\lambda s}\right)(u)-(U n)(u)\right] t^{-1}} \\
& =-i \lambda(2 \pi)^{-1 / 2} \int_{-\infty}^{\infty} \frac{f(v)}{(v-i+i \lambda t)(v-i)} \exp (-i v u) d v \\
& =-i \lambda(2 \pi)^{-1 / 2} \int_{-\infty}^{\infty}(U n)(v) U_{y}\left[\frac{1}{y-i+i \lambda t}\right](u-v) d v \\
& =\lambda \int_{u}^{\infty}(U n)(v) \exp (1-\lambda t)(u-v) d v .
\end{aligned}
$$

Hence by the Fubini theorem we see that

$$
\begin{gathered}
(2 \pi)^{-1 / 2} \int_{-\infty}^{0}\left(\left(U f_{\lambda t}\right)(u)-(U n)(u)\right) t^{-1} \exp (\lambda t u+i z u) d u \\
=\lambda(2 \pi)^{-1 / 2} \int_{-\infty}^{0}\left[\int_{u}^{\infty}(U n)(v) \exp (-1+\lambda t) v d v\right] \exp (1+i z) u d u \\
=\lambda(2 \pi)^{-1 / 2} \int_{-\infty}^{0}(U n)(v) \exp (-1+\lambda t) v d v \int_{-\infty}^{v} \exp (1+i z) u d u \\
+\lambda(2 \pi)^{-1 / 2} \int_{0}^{\infty}(U n)(v) \exp (-1+\lambda t) v d v \int_{-\infty}^{0} \exp (1+i z) u d u \\
=-\frac{i \lambda}{z-i}(2 \pi)^{-1 / 2}\left[\int_{-\infty}^{0}(U n)(u) \exp (\lambda t+i z) v d v\right. \\
\left.\quad+\int_{0}^{\infty}(U n)(v) \exp (-1+\lambda t) v d v\right]
\end{gathered}
$$

From this equality it follows that

$$
\left[\int_{-\infty}^{\infty} \mid(2 \pi)^{-1 / 2} \int_{-\infty}^{0}\left(\left(U f_{\lambda t}\right)(u)-(U n)(u)\right) t^{-1} \exp (\lambda t u+i z u) d u\right.
$$




$$
\begin{aligned}
& +\frac{i \lambda}{z-i} \operatorname{lit}_{a \rightarrow \infty}(2 \pi)^{-1 / 2} \int_{-a}^{0}(U n)(u) \exp (i z u) d u \\
& \left.+\left.\frac{i \lambda}{z-i}(2 \pi)^{-1 / 2} \int_{0}^{\infty}(U n)(u) \exp (-u) d u\right|^{2} d z\right]^{1 / 2} \\
& =|\lambda|\left[\int_{-\infty}^{\infty} \frac{1}{z^{2}+1}(2 \pi)^{-1} \mid-\int_{-\infty}^{0}(U n)(u) \exp (\lambda t+i z) u d u\right. \\
& -\int_{0}^{\infty}(U n)(u) \exp (-1+\lambda t) u d u+\operatorname{lin}_{a \rightarrow \infty} \int_{-a}^{0}(U n)(u) \exp (i z u) d u \\
& \left.+\left.\int_{0}^{\infty}(U n)(u) \exp (-u) d u\right|^{2} d z\right]^{1 / 2} \longrightarrow 0 \text { as } t \longrightarrow+0 .
\end{aligned}
$$

Also by Plancherel's theorem we see that

$$
\begin{aligned}
& {\left[\int_{-\infty}^{\infty} \mid\left\{(2 \pi)^{-1 / 2} \int_{-\infty}^{0}(U n)(u) \exp (\lambda t u+i z u) d u\right.\right.} \\
& \left.-\lim _{a \rightarrow \infty}(2 \pi)^{-1 / 2} \int_{-a}^{0}(U n)(u) \exp (i z u) d u\right\} t^{-1} \\
& \left.+\left.\lambda \operatorname{limim}_{a \rightarrow \infty}(2 \pi)^{-1 / 2} \int_{-a}^{0}|u|(U n)(u) \exp (i z u) d u\right|^{2} d z\right]^{1 / 2} \\
& =\left[\int_{-\infty}^{0}\left|(U n)(u)(\exp (\lambda t u)-1) t^{-1}-\lambda\right| u|(U n)(u)|^{2} d u\right]^{1 / 2} \longrightarrow 0 \text { as } t \longrightarrow+0 .
\end{aligned}
$$

Hence from (2.10) we obtain that (the first term of (2.9)) $\rightarrow 0$ as $t \rightarrow+0$. In the same manner we can prove that (the second term of (2.9)) $\rightarrow 0$ as $t \rightarrow+0$. It is easily seen that (the third term of (2.9)) $\rightarrow 0$ as $t \rightarrow+0$. From these facts it follows that $A_{\lambda} f$ $=B_{i} f$.

Q.E.D.

Theorem 2.2. The infinitesimal generator $A_{-i q}$ of the group $\left\{P_{-i q}(t)\right.$ : $-\infty<t<\infty\}$ in Theorem 1.2 is given by the following form;

$$
\begin{gathered}
D\left(A_{-i q}\right)=\left\{f \in W^{2}:|u|(U n)(u) \in L^{2}(R)\right\} \text { and for } f \text { in } D\left(A_{-i q}\right) \\
\begin{aligned}
\left(A_{-i q} f\right)(z)=q & \lim _{a \rightarrow \infty}(2 \pi)^{-1 / 2} \int_{-a}^{a}(U n)(u)(\operatorname{sign} u) \exp (i z u) d u \\
& +i q(z-i) \operatorname{lijim}_{a \rightarrow \infty}(2 \pi)^{-1 / 2} \int_{-a}^{a}|u|(U n)(u) \exp (i z u) d u .
\end{aligned}
\end{gathered}
$$

Proop. In the same manner as the proof of Theorem 2.1 we obtain

$$
D\left(A_{-i q}\right)=\left\{f \in W^{2}:|u|(U n)(u) \in L^{2}(R)\right\} .
$$

If $f$ is in $D\left(A_{-i 4}\right)$, then

$$
n(u)=(u-i)(U n)(u) \cdot \frac{1}{u-i} \in L^{1}(R) .
$$

By the Parseval theorem we see that 


$$
\begin{aligned}
t^{-1}\left(U f_{-i q t}-U n\right)(u) & =-(2 \pi)^{-1 / 2} q \int_{-\infty}^{\infty} \frac{f(v)}{(v-i)(v-i+q t)} \exp (-i u v) d v \\
& =-(2 \pi)^{-1 / 2} q \int_{-\infty}^{\infty}(U n)(w) U_{v}\left[\frac{1}{v-i+q t}\right](u-w) d w \\
t^{-1}\left(U k_{-i q t}-U n\right)(u) & =(2 \pi)^{-1 / 2} q \int_{-\infty}^{\infty} \frac{f(v)}{(v-i)(v-i-q t)} \exp (-i u v) d v \\
& =(2 \pi)^{-1 / 2} q \int_{-\infty}^{\infty}(U n)(w) U_{v}\left[\frac{1}{v-i-q t}\right](u-w) d w
\end{aligned}
$$

Since

$$
(U n)(w), U_{v}\left[\frac{1}{v-i+q t}\right](w), U_{v}\left[\frac{1}{v-i-q t}\right](w)
$$

are in $L^{1}(R),(2.12)$ and (2.13) are functions in $L^{1}(R)$ and in $L^{2}(R)$. From these facts and from the same argument as the proof of Theorem 2.1 we obtain (2.11).

Q.E.D.

Note that if $f$ is in $D\left(A_{-i q}\right)$,

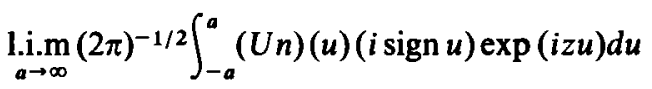

$$
\begin{aligned}
& =(2 \pi)^{-1 / 2} \int_{-\infty}^{\infty}(U n)(u)(i \operatorname{sign} u) \exp (i z u) d u .
\end{aligned}
$$

ReMARK 2.1. In Theorem 2.1 and Theorem 2.1

1) $\quad \frac{1}{\pi} P \int_{-\infty}^{\infty} \frac{n(u)}{u-z} d u=(2 \pi)^{-1 / 2} \int_{-\infty}^{\infty}(U n)(u)(i \operatorname{sign} u) \exp (i z u) d u$,

2) if $n(u)$ is absolutely continuous on each bounded closed interval and $n^{\prime}(u)$ is absolutely integrable over $R$,

$$
\frac{1}{\pi} P \int_{-\infty}^{\infty} \frac{n^{\prime}(u)}{u-z} d u=-\lim _{a \rightarrow \infty}(2 \pi)^{-1 / 2} \int_{-a}^{a}|u|(U n)(u) \exp (i z u) d u
$$

Here

$$
\frac{1}{\pi} P \int_{-\infty}^{\infty} \frac{n(u)}{u-z} d u, \frac{1}{\pi} P \int_{-\infty}^{\infty} \frac{n^{\prime}(u)}{u-z} d u
$$

denote the Hilbert transforms of $n(u)$ and $n^{\prime}(u)$.

\section{References}

[1] N. I. Achieser, Theory of Approximation, Frederick Ungar Publishing Co., New York. [2] R. R. Coifman and C. Fefferman Weighted norm inequalities for maximal functions and 
singular integrals, Studia Math. 51 (1974), 241-250.

[3] N. Dunford and J. T. Schwartz, Linear operators, Part 11: Spectral theory, Pure and Appl. Math. Vol. 7 (Interscience, New York, 1963).

[4] E. Hille and E. S. Pphillips, Functional analysis and semigroups, A.M.S. Colloq. Publ., Vol. 31 (1957).

[ 5] S. Koizumi, On the singular integrals. V, Proc. Japan Acad. 35 (1959), 1-6.

[6] - On the singular integrals. VI, Proc. Japan Acad. 35 (1959), 323-326.

[ 7] - On the Hilbert transform I, Hokkaido Math. J. (J. Faculty Sci. Hokkaido Univ.)16 (1959), 153-224.

[8] K. Takano, An analogous method to Cameron and Storvick's function space integral and evolution systems, J. London Math. Soc. 16 (1977), 83-95.

[9] E. C. Titchmarsh, Introduction to the theory of the Fourier integrals, Oxford University Press, Second Edition, 1948.

[10] K. Yosida, Functional analysis, Springer-Verlag, Second Edition, 1968.

[11] N. Wiener, The Fourier integral and certain of it application, Dover Publishing. 